\title{
Low Cost S-band Beamsteering Antennas for Long Range Professional UAV
}

\author{
P. Teillet ${ }^{1,2}$, M. Thevenot ${ }^{2}$, C. Menudier ${ }^{2}$, P-Y. Fulchiron ${ }^{1}$ \\ ${ }^{1}$ DELAIR, 676 rue Max Planck, 31670 Labège \\ ${ }^{2}$ Univ. Limoges, CNRS, XLIM, UMR 7252, F-87000 Limoges, France \\ paul.teillet@delair.aero
}

\begin{abstract}
This work presents an effective solution of low-cost beamsteering antennas for professional UAV. The concept of parasitic element antenna has been revisited to meet integration and industrialization specifications. The originality of this work is based on the co-design of the radiating array and the phase shifters. These reactive loads are modelized and used in a synthesis process to optimize the efficiency of the antenna in directions of interest. Two prototypes were manufactured and measured.

Keywords - beamsteering antenna, parasitic element antenna, UAV antenna, integrated reflection-type phase shifters, low cost, beam steering $\mathrm{S}$ band.
\end{abstract}

\section{INTRODUCTION}

Professionals Unmanned Aerials Vehicle (UAV) for industry are becoming essentials for topographic studies, surveillance operations or aerial imagery. These competitive markets enforce the needs for efficiency and endurance flight to achieve long range autonomous operation. Even though drones are equipped with an autopilot allowing execution of a programmed flight plan, a command and control link with a ground station is required to safely conduct operations without endangering people or other aircraft. The reliability of this radio link is necessary and mustn't be broken. Because of size and weight restrictions, most UAV use omnidirectional antennas to achieve this radio link regardless of the yaw and pitch axis of the aircraft. However, low gain provided by these antennas limits the command \& control link and therefore limits the drone's operations range. To achieve more reliable link and to extend range of operations, these omnidirectional antennas can be replaced by higher gain beamsteering antennas. However, these antennas must satisfy specifics restrictions such as ease of integrations, low weight, energy efficient and low-cost manufacturing. In this context, the DELAIR drone manufacturer would like to equip some of their UAV with a beamsteering antenna (Fig. 1) to improve a S-band radio link.

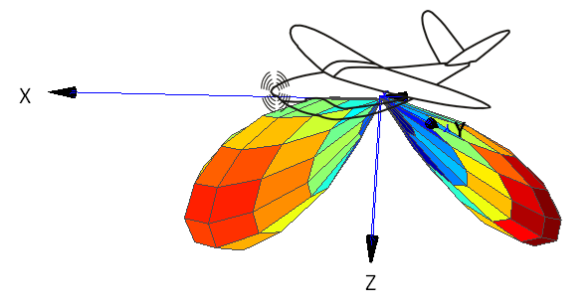

Fig. 1. High gain antenna for range enhancement
Beamsteering is usually achieved by phased array antenna including expensive and power consuming electronics such as integrated phase shifters [1]. Moreover, these chips and the feeding network can exhibit significant RF losses that are often greater than several dB. Due to the technological complexity and costs, and the achievable performances, a phased array antenna architecture doesn't offer the best trade-off for an UAV integration.

This work presents a parasitic element antenna (PEA) architecture, more suited to meet the UAV specifications. Lots of academic papers deals with such antenna concept with a moderate number of parasitic elements and switches to realize diagram diversity [2][3]. To our knowledge, we don't know any industrial application for on-board beamsteering mission with this kind of antenna.

In order to obtain continuous beamsteering, the parasitic elements must be connected to tunable reactive loads, as proposed in the Harrington's description [4] shown in Fig. 2. This antenna's concept was already prototyped in [5][6].

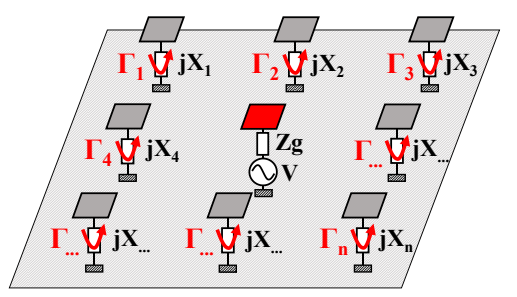

Fig. 2. A nine-element array with 8 parasitic elements

\section{ANTENNA DESIGN}

\section{A. Legacy Solutions from Previous Designs}

Reconfigurable Parasitic Element Antenna (RPEA) design can take advantages of the lack of feed network to freely change the number of elements and the shape of the radiating panel. These antennas are composed of two types of elements : ones are radiating active elements and the others are parasitic elements excited by mutual couplings.

In previous works [5][6], we demonstrated the ability to deal with several tens of parasitic monopoles managing the beamsteering. A set of SMA connectable and controllable lowcost reactive loads were emulated by coplanar waveguide (CPW) loaded with varactor diodes (Fig. 3). In this previous demonstrator, every monopole of the radiating panel was 
equipped with a SMA connector too in order to enable the scattering matrix measurement of the monopole array.

The beamsteering needed to define the DC bias voltage for each reactive load. Due to the high number of parasitic elements, it was necessary to resort to optimization algorithm [7] using realistic models (e.g. measured) for both the controllable reactive loads and the antenna S-matrix. The knowledge of these models was made possible thanks to the SMA connectors used in the demonstrator shown in Fig. 3.
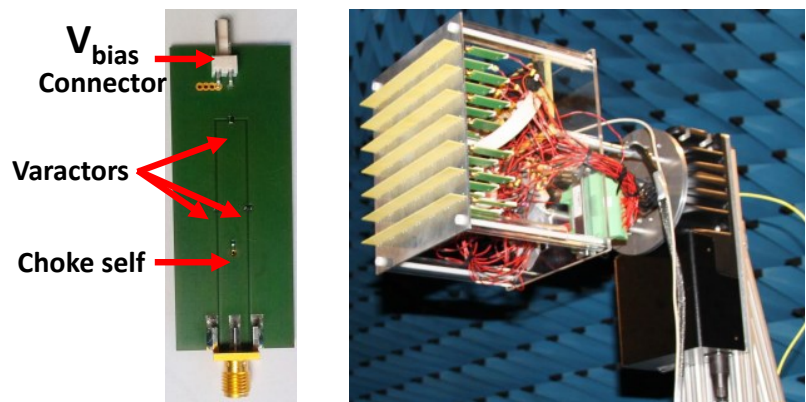

Fig. 3. SMA connected tunable reactive load (left) and first 49 elements RPEA demonstrator (right) [6]

This previous prototype provided very good results, but it appears too heavy and bulky for an UAV integration.

\section{B. Design Evolution for UAV Integration}

The originality of this work lies in the interconnection (SMA) removal between the parasitic elements and the reactive loads. This means that direct EM characterizations will become impossible. Fig. 4 show the integration of a monopole with a reflection-type phase shifter soldered together on a single PCB. These monopoles are now small brass pipes plugged in the PCB The transition between the monopoles and the phase shifters were optimized to enhance the coupling with the grounded coplanar waveguide (GCPW) mode.

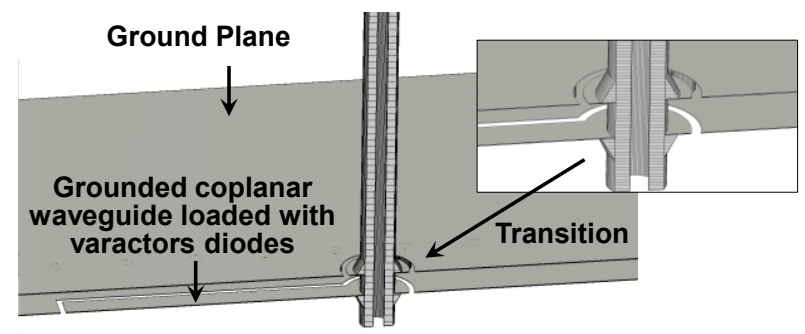

Fig. 4. Integrated reflection-type phase shifter and radiating element transition

A full-wave simulation is the only way to get a S-matrix model for the monopole array. However, the extraction of the controllable reactive load model has required the development of a specific measurement kit [8] that has provided the characteristics shown in Fig. 5.
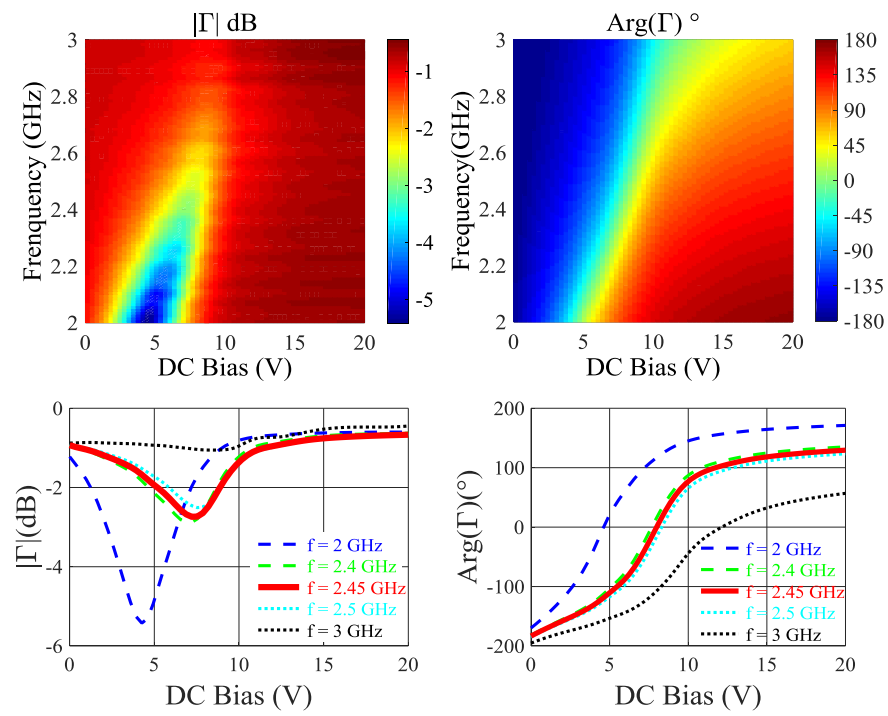

Fig. 5. Integrated reflection-type phase shifter model

To optimize the efficiency of the antenna, the dimensions of the phase shifter were set to limit the insertion losses below 2.5 $\mathrm{dB}$, in return the phase excursion will be only $310^{\circ}$ at $2.45 \mathrm{GHz}$.

\section{Radiating Arrays}

The absence of feeding network is an advantage to adjust the antenna shape to the integration requirements. There is no restriction on the number of elements, array size, elements locations, etc... For every antenna array shown in Fig. 6, there is only one active element fed in the middle of the array. The only requirement is to enhance the mutual couplings between antennas. Hence, the array grid step is set to $\lambda_{0} / 4$ at $2.45 \mathrm{GHz}$. Fig. 6 shows three different array arrangements with their respective performances compared in Table 1.

Table 1. Examples of realized gain toward horizon.

\begin{tabular}{|c|c|c|}
\hline Array & $\begin{array}{c}\text { Mean Realized } \\
\text { Gain }\left(\boldsymbol{\theta}^{=90^{\circ}}\right)\end{array}$ & $\begin{array}{c}\text { Roll-off } \\
\text { (azimut) }\end{array}$ \\
\hline $3 \times 3$ square grid & $5.1 \mathrm{~dB}$ & $1.1 \mathrm{~dB}$ \\
\hline $5 \times 5$ square grid & $7.1 \mathrm{~dB}$ & $0.7 \mathrm{~dB}$ \\
\hline 19 elements triangular grid & $6.7 \mathrm{~dB}$ & $1.4 \mathrm{~dB}$ \\
\hline
\end{tabular}

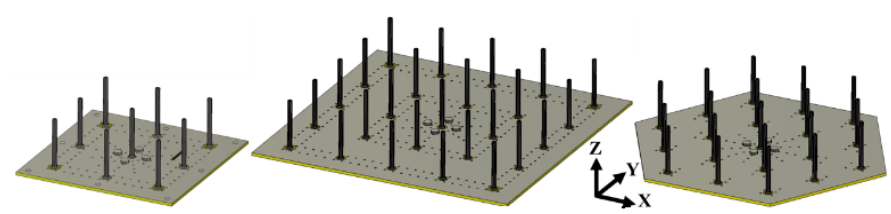

Fig. 6. Examples of possible array

In the next sections, we will only detail the performances of the $3 \times 3$ and $5 \times 5$ elements square grid arrays.

\section{Antennas Prototyping \& MEASUREMENTS}

Two square grid array antennas were manufactured. They are shown in Fig. 9. The PCB substrate is a FR4 Isola IS400 (@) $500 \mathrm{MHz}: \varepsilon r=3.9$ and $\tan (\delta)=0.022)$. Fig. 7 is a picture of the backside antenna PCB, showing the integrated phase shifters with varactor diodes (Skyworks SMV2202) routed to 
their DC bias voltage connector. The only active monopole is fed through an SMA connector.

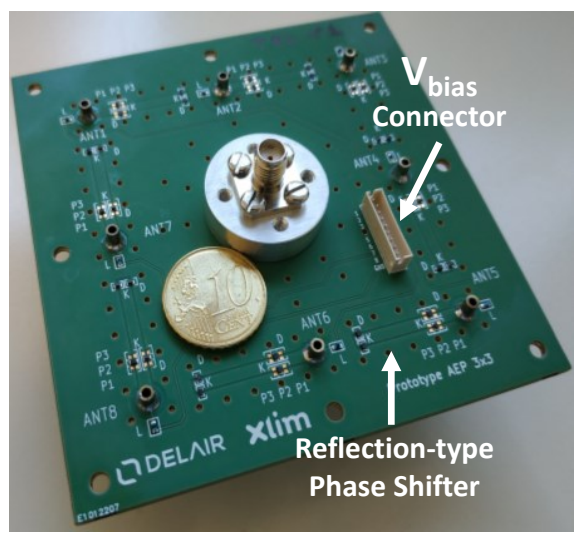

Fig. 7. Backside of the manufactured $3 \times 3$ RPEA

The DC bias voltages definition consist to maximize the realized gain in a given direction. Hence, an algorithm [7] optimizes the total efficiency of the antenna, minimizing the losses in the phase shifters and producing a good impedance matching. Results of these synthesis process are shown in Table 2.

Table 2. Simulated result for the presented cases

\begin{tabular}{|c|c|c|c|c|}
\hline \multirow{2}{*}{ Antenna } & $\begin{array}{c}\text { Elevation } \\
(\boldsymbol{\Theta})\end{array}$ & $\begin{array}{c}\text { Azimut } \\
(\boldsymbol{\phi})\end{array}$ & $\begin{array}{c}\text { Realized } \\
\text { Gain }\end{array}$ & $\begin{array}{c}\text { Total } \\
\text { efficiency }\end{array}$ \\
\hline \multirow{2}{*}{$\mathbf{5} \mathbf{5} 5$} & \multirow{2}{*}{$90^{\circ}$} & $0^{\circ}$ & $7 \mathrm{~dB}$ & $56 \%$ \\
\cline { 3 - 5 } & \multirow{2}{*}{$\mathbf{3} \mathbf{3}$} & $45^{\circ}$ & $7.1 \mathrm{~dB}$ & $59 \%$ \\
\cline { 3 - 5 } & \multirow{2}{*}{$90^{\circ}$} & $0^{\circ}$ & $4.9 \mathrm{~dB}$ & $79 \%$ \\
\cline { 3 - 5 } & $45^{\circ}$ & $5.4 \mathrm{~dB}$ & $82 \%$ \\
\hline
\end{tabular}

Both antennas were successfully measured for different beamsteering angle and compared to the simulations (Fig. 8). The cyan and magenta markers are experimental gains for several azimuthal angles towards the horizon. The measured realized gain is consistent with the simulations for the $3 \times 3$ RPEA (blue solid line) and the 5x5 RPEA (red solid line).

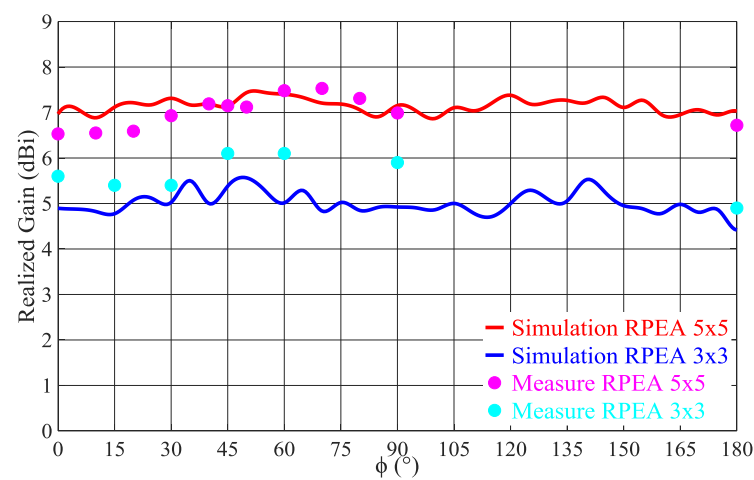

Fig. 8. Comparison of the realized gain toward horizon $\left(\theta=90^{\circ}\right)$ for the two RPEA at frequency $=2.45 \mathrm{GHz}$

A few configurations were selected to assess the good agreement between the measurements and the antennas numerical models. Fig. 10 exhibits antennas matchings and Fig. 11 shows the comparisons of radiation patterns.

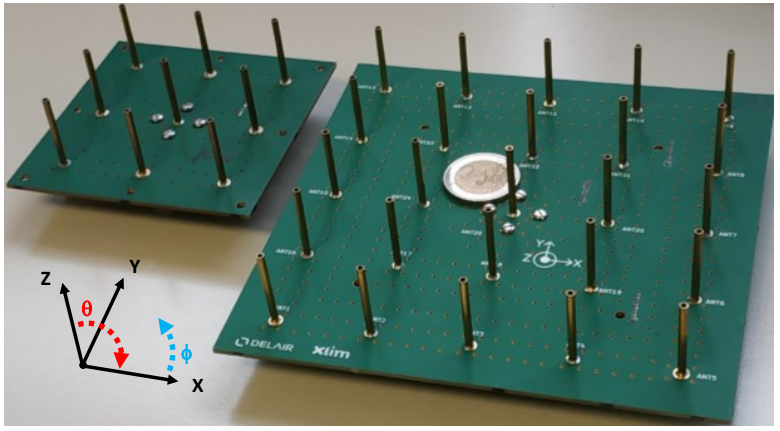

Fig. 9. Frontside of the manufactured 3x3 RPEA (left) and the 5x5 RPEA (right)
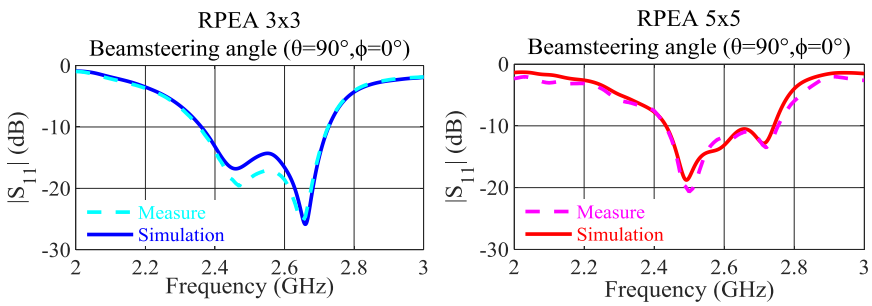

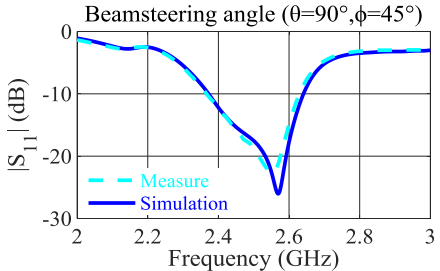

(a)

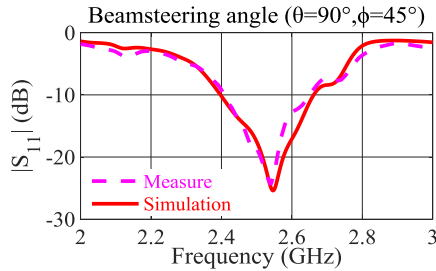

(b)
Fig. 10. Reflection coefficient on the fed port of the $3 \times 3$ RPEA (a) and the $5 \times 5$ RPEA (b) for two beamsteering angle $\left\{\theta_{0}, \phi_{0}\right\}:\left\{90^{\circ}, 0^{\circ}\right\}$ and $\left\{90^{\circ}, 45^{\circ}\right\}$

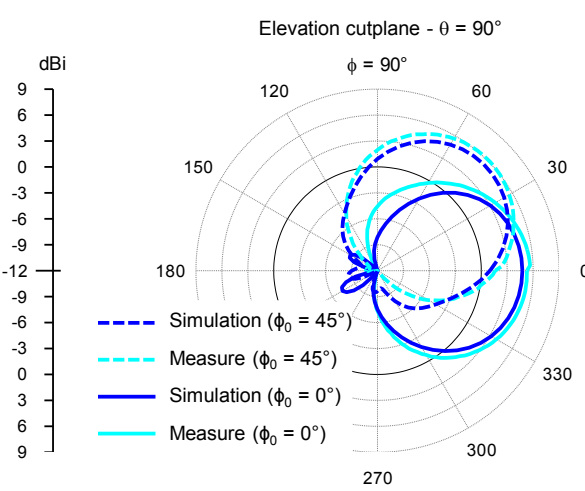

(a)

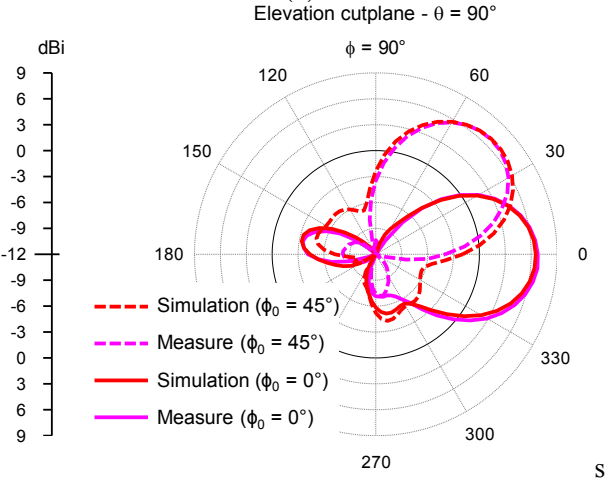

(b)

Fig. 11. Realized gain at $2.45 \mathrm{GHz}$ for to beamsteering angle $\{\theta 0, \phi 0\}:\left\{90^{\circ}\right.$, $\left.0^{\circ}\right\}$ and $\left\{90^{\circ}, 45^{\circ}\right\}$. (a) RPEA $3 \times 3$ (b) RPEA $5 \times 5$ 
The good agreements between simulations and measurements demonstrate the accuracy of the numerical models for the phase shifters and the antennas, used in the synthesis algorithm. Therefore, we can rely on these models to optimize the whole DC bias voltages tables. It has been checked that these realized gains level can be achieved whatever the azimuthal direction.

\section{CRITICAL ANALYSIS}

In this section, the global efficiency of each antenna is analyzed, and we estimate whether a low loss RF substrate can significantly reduce the losses.

First, the total efficiencies of both antennas were deduced from the simulated gains and directivities and shown in Fig.12. The efficiency for the $3 \times 3$ RPEA reaches about $80 \%$ while the $5 \times 5$ one is only $60 \%$. We conclude that the number of parasitic elements directly increases losses.

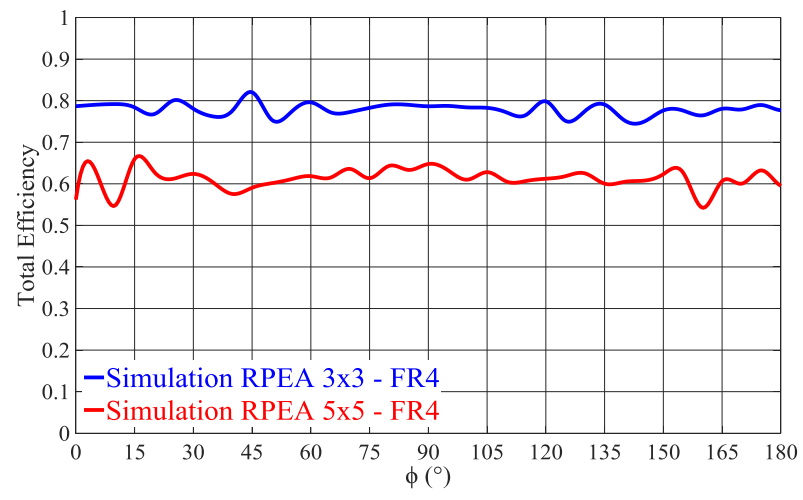

Fig.12. Comparison of the simulated total efficiency toward horizon $\left(\theta=90^{\circ}\right)$ for the two RPEA at frequency $=2.45 \mathrm{GHz}$

Although the losses in the 5x5 RPEA are greater, this antenna has a higher gain (Fig. 8). Hence, the 5x5 RPEA appeared better for our application.

The FR4 substrate was initially chosen to meet the antenna cost objective but it can be possible to improve the $5 \times 5$ RPEA performances by using a RF-suitable substrate. This will be now evaluated.

A new simulation was proceeded in which dielectric losses were set to $\tan (\delta)=0.003$. Fig. 13 compares the directivities (dashed line) and the realized gains (solid line) for both substrates (FR4 vs low-loss substrate) and for the same DC bias voltages laws applied. The simulated directivities of both antennas are comparable but thanks to the low-loss substrate, the realized gain is enhanced by only $0.7 \mathrm{~dB}$ to be greater than $7.5 \mathrm{~dB}$. Therefore, the residual losses $(1.5 \mathrm{~dB}$ between directivity and gain) are mainly due to the SMD varactors diodes. We concluded that the use of an expensive, low-loss RF substrate offers too few improvements in return for the additional cost, so a $-2 \mathrm{~dB}$ efficiency is a proper trade-off for the UAV application.

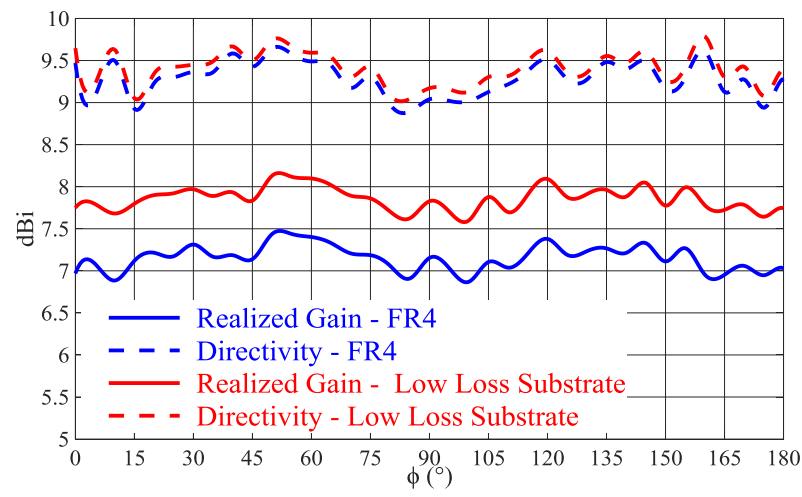

Fig. 13. Comparison between a standard FR4 5x5 RPEA and a low loss substrate $5 \times 5$ RPEA toward the horizon $\left(\theta=90^{\circ}\right)$ at frequency $=2.45 \mathrm{GHz}$

\section{CONCLUSION}

This work focuses on a simplified architecture of low cost integrated steerable antennas, for professional long-range UAV. They are parasitic elements antennas with reactive loads, or reflection-type phase shifters, incorporating low cost varactor diodes tuned by DC bias voltages. The array antenna and the phase shifters are co-designed on an unique low cost PCB (FR4). The DC bias voltage laws are defined for thousands beamsteering angles using accurate numerical models for both the monopole array and the reflection-type phase shifters. The method optimizes the gain for each direction, reducing the phase shifters losses and the input matching. The experimental characterizations are consistent with the simulations results. The 5x5 RPEA exhibits good performances and is compliant with the UAV applications.

\section{ACKNOWLEDGMENT}

The authors would like to thank E. Arnaud for the time he spent to measure the antenna in anechoic chamber.

\section{REFERENCES}

[1] R. J. Mailloux, "Phased Array Antenna Handbook", 2nd edition. Artech House Publishers, 2005

[2] T. Ohira,K. Gyoda, "Electronically Steerable Passive Array Radiator Antennas for Low-Cost Analog Adaptive Beamforming", IEEE Internarional Conference on Phased Array Systems and Technology, $101-104,2000$

[3] R. Vaughan, "Switched parasitic elements for antenna diversity,", IEEE Transactions on Antennas and Propagation, vol. 47, no 2, p. 399-405, 1999

[4] R. F. Harrington,"Reactively controlled directive arrays," IEEE Trans. Antennas Propag., vol. 26, n³, pp. 390-395, Mai 1978.

[5] F. Fezai et al, "Reconfigurable Parasitic Element Antenna Using Reflection Phase Shifters", IEEE Antennas and Wireless Propagation Letters, 2014.

[6] C. Menudier et al, Menudier, C., et al. "Synthesis of a 2D reconfigurable parasitic element antenna.", Microwave Conference (EuMC), 2016 46th European. IEEE, 2016.

[7] M. Thevenot et al, "Synthesis of Antenna Arrays and Parasitic Antenna Arrays with Mutual Couplings," International Journal Antennas Propag., vol. 2012.

[8] R. B .Marks, "A multiline method of network analyzer calibration". IEEE Transactions on Microwave Theory and Techniques, vol. 39, no 7, pp. 1205-1215, 1991 